\title{
Architecting Neonicotinoid-Scavenging Nanocomposite Hydrogels for Environmental Remediation
}

Abdulaziz Alammar, ${ }^{a}$ Sang-Hee Park, ${ }^{\mathrm{b}}$ Izwaharyanie Ibrahim, ${ }^{\mathrm{c}}$ Deepak Arun, ${ }^{\mathrm{d}}$ Tibor Holtzl,,${ }^{\mathrm{e}}$ Ludovic F. Dumée, ${ }^{\mathrm{g}}$ Hong Ngee Lim, ${ }^{\mathrm{c}}$ Gyorgy Szekely,b,*

${ }^{a}$ Department of Chemical Engineering \& Analytical Science, The University of Manchester, Sackville street, The Mill, Manchester, M1 3BB, United Kingdom

${ }^{\mathrm{b}}$ Advanced Membrane and Porous Materials Center, King Abdullah University of Science and Technology (KAUST), Thuwal, 23955-6900, Saudi Arabia

${ }^{c}$ Department of Chemistry, Faculty of Science, Universiti Putra Malaysia, 43400 UPM Serdang, Selangor, Malaysia

${ }^{\mathrm{d}}$ Saveetha School of Engineering, Saveetha Institute of Medical and Technical Sciences, Chennai, Tamil Nadu, 602105, India

${ }^{\text {e }}$ MTA-BME Computation Driven Chemistry Research Group, Department of Inorganic and Analytical Chemistry, Budapest University of Technology and Economics, Muegyetem rkp. 3., Budapest 1111, Hungary

${ }_{\mathrm{f}}^{\mathrm{f}}$ Furukawa Electric Institute of Technology, Kesmark utca 28/A, Budapest 1158, Hungary

${ }^{g}$ Deakin University, Geelong, Institute for Frontier Materials, Waurn Ponds, 3216, Victoria, Australia

*Corresponding author: gyorgy.szekely@kaust.edu.sa; www.szekelygroup.com; +966 128082769

\section{Highlights}

- Removal of neonicotinoids from aqueous solutions, including groundwater, down to $0.1 \mu \mathrm{g} \mathrm{L}^{-1}$.

- Robust cellulose acetate-based hydrogels were prepared in Cyrene and MeTHF green solvents.

- The synergistic effect of PIM-1 and GO in the nanocomposite was investigated by molecular dynamics.

- Sustainable regeneration of hydrogels using ultrasound, recovering virtually $100 \%$ of the adsorption capacity.

- Continuous adsorption process successfully treated groundwater from the Adyar river in Tamil Nadu, India.

\section{Keywords}

Neonicotinoids, Water purification, Green solvents, Polymers of intrinsic microporosity, Graphene

\section{Graphical abstract}

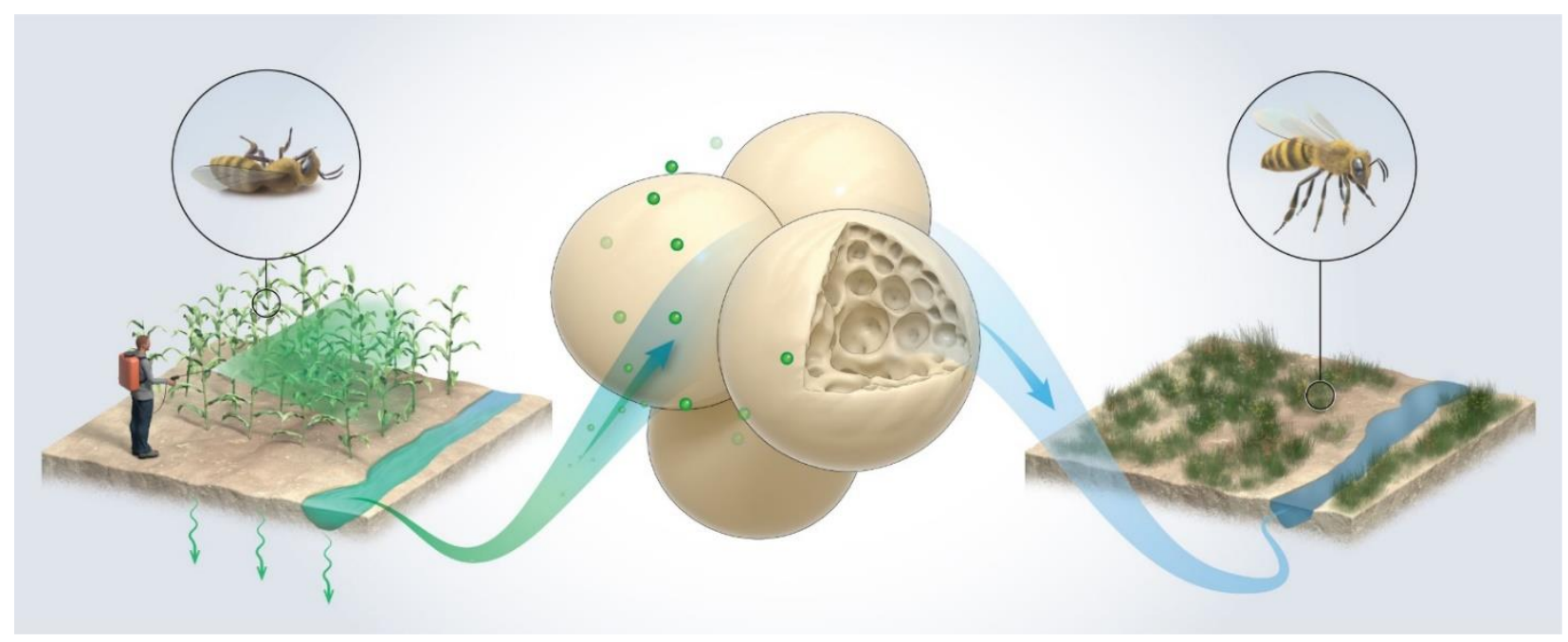




\begin{abstract}
The ubiquitous presence of neonicotinoid insecticides in the environment poses potential health concerns across all biomes, aquatic systems, and food chains. This global environmental challenge requires robust, advanced materials to efficiently scavenge and remove these harmful neonicotinoids. In this work, we engineered nanocomposite hydrogels based on sustainable cellulose acetate for water treatment. The nanocomposite hydrogels were incorporated with small quantities of polymers of intrinsic microporosity (PIM-1) and graphene oxide (GO). We prepared the hydrogels using green solvents such as Cyrene and MeTHF via simple dropwise phase inversion. High adsorption capacity and fast kinetic behavior toward acetamiprid, clothianidin, dinotefuran, imidacloprid, and thiamethoxam were observed. We also developed a rapid and sustainable ultrasound-assisted regeneration method for the hydrogels. Molecular dynamics of the complex quaternary system revealed the synergistic effects of the components, and the presence of PIM1 was found to increase the GO surface area available for neonicotinoid scavenging. We demonstrated the robustness and practicality of the nanocomposites in continuous environmental remediation by using the hydrogels to treat contaminated groundwater from the Adyar river in India. The presented methodology is adaptable to other contaminants in both aqueous environments and organic media.
\end{abstract}

\title{
1. Introduction
}

The use of pesticides for agriculture and crop production is increasing globally, according to the Food and Agriculture Organization of the United Nations (FAO) [1]. Recently, environmental regulations across the agricultural sector have become more stringent to minimize greenhouse emissions and help to reach the Sustainable Development Goals as part of the Paris Agreement on Climate Change. Neonicotinoids are chemically related to nicotine, and they were developed in the 1990s as alternative pesticides to replace organochlorines, organophosphates, and carbamates [2]. They are utilized globally by approx. 120 countries and account for $25 \%$ of the agriculture insecticide market [2,3]. Studies have shown that up to $98 \%$ of neonicotinoids remain in the soil as residuals due to their water solubility and high persistence, usually ending up in groundwater or surface waters (Fig. 1) [4].

Neonicotinoids are systemically absorbed through plant tissue to reach the nectar and pollen of the treated crops, leading to the potential exposure of pollinators, especially wild bees and honeybees. In recent years, debates and uncertainty have arisen regarding the use and environmental effects of neonicotinoids. Abandoning neonicotinoids could cause more damage to the agriculture sector due to the lack of effective alternatives [5], even though neonicotinoids negatively impact vital pollinators such as bees [6-11]. In 2013, the European Union (EU) banned the use of three neonicotinoids, namely, clothianidin, imidacloprid, and thiamethoxam, on flowering crops, and these restrictions were extended to all outdoor use in late 2018 $[12,13]$. In addition, dinotefuran was also classified as highly toxic to honeybees by the International Union 
of Pure and Applied Chemistry (IUPAC) [14]. Nevertheless, the use of neonicotinoids in most countries has not yet been sufficiently curtailed. Thus, neonicotinoids are increasingly ubiquitous in the environment owing to their widespread use and high solubility in water. Apart from directly affecting pollinators, traces of neonicotinoids have been found in honey, fruit, vegetables, milk, drinking water, and shrimp, among others [15-18]. Recent studies have linked neonicotinoids to negative environmental impacts on soil and water resources, with concentrations of neonicotinoids found in the range of $\mu \mathrm{g} \mathrm{L}^{-1}$ in vital surface and groundwater resources. Such issues have motivated research in recent years to find an engineering solution for public water systems [19].

Adsorption is the most widely used technology for the removal of pesticides from the aqueous environment because it is economically efficient and environmentally friendly [20]. Powder-like adsorbents have practical limitations in their handling and require complex processes to achieve efficient recovery from water. Spherical-shape hydrogels made from polysaccharide-based materials such as cellulose, chitosan, and sodium alginate fabricated via a simple phase-separation technique have been proposed as a convenient and practical solution [21]. In this work, we have chosen cellulose acetate as the polymer matrix to develop practical core-shell hydrogel spheres using the phase-separation technique.

Hydrogels are widely used in agriculture for retaining water, improving soil permeability, and reducing irrigation dependence, as well as reducing the need to use hormones, fertilizers, and pesticides [22,23]. Hydrogels have several unique and beneficial characteristics, including a high swelling degree and adsorption capacity. However, they need to be mechanically robust and reusable to make them a feasible technology [20,24,25]. This can be achieved by architecting nanocomposites that incorporate highperformance polymers and advanced materials (Fig. 1) [26,27]. Polymers with intrinsic microporosity have been widely studied for numerous applications, including water treatment and adsorption, and in particular for the removal of antibiotics and dyes [28-30]. Such polymers have vacant spaces between the polymer chains because they are not packed due to the contortion of the fused rings, thus giving the polymers high free volumes. Graphene oxide (GO) has also been successfully employed in adsorptive water treatment and photocatalysis [31-35]. The objective of this work is to investigate the synergistic effect of GO and polymers of intrinsic microporosity (PIM-1), combined with cellulose acetate (CA) hydrogel for the removal of neonicotinoids from the aqueous environment. The novel materials were applied in continuous water purification facilitated by ultrasound-assisted regeneration of the hydrogels. 


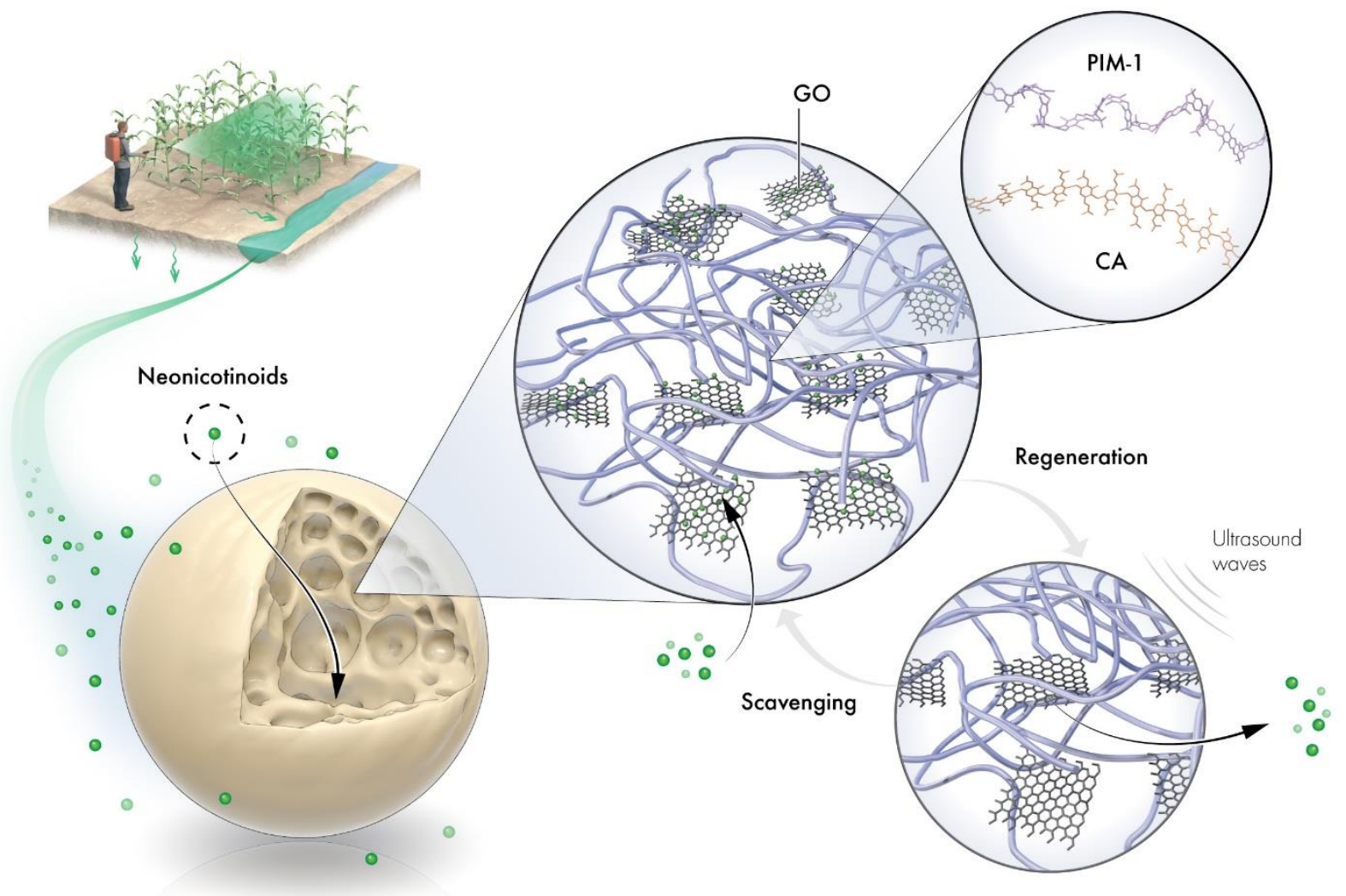

Fig. 1 Schematic representation of neonicotinoid contamination of waterways and their environmental clean-up using nanocomposites hydrogels composed of graphene oxide (GO), polymers of intrinsic microporosity (PIM-1), and cellulose acetate (CA). Sustainable regeneration of the hydrogels via ultrasonication.

\section{Material and methods}

\subsection{Material}

5,5',6,6'-Tetrahydroxy-3,3,3',3'-tetramethyl-1,1'-spirobisindane (TTSBI, 98\%, Synthon Chemicals) was dissolved in methanol, re-precipitated from dichloromethane, and dried under vacuum at room temperature before use. Tetrafluoroterephthalonitrile (TFTPN, 98\%, Chemos) was used as received. Anhydrous $\mathrm{K}_{2} \mathrm{CO}_{3}$ (99\%, Fisher Scientific) was ground to a fine powder and dried in a vacuum oven at $110{ }^{\circ} \mathrm{C}$ overnight before use. The cellulose acetate (CA) was purchased from BDH Chemical Ltd (Malaysia). 2methyltetrahydrofuran (MeTHF), and Cyrene ${ }^{\circledR}$ were purchased from Sigma-Aldrich and used as received. Tetrafluoroterephthalonitrile, $\quad 5,5^{\prime}, 6,6^{\prime}$-tetrahydroxy-3,3,3',3'-tetramethyl-1,1'-spirobisindane, $\quad N, N$ dimethylacetamide (DMAc) and $p$-cymene were purchased from Alfa-Aesar. All aqueous solutions were prepared using Milli-Q Type II water with a resistivity of $18.2 \mathrm{M} \Omega \mathrm{cm}$ at $25^{\circ} \mathrm{C}$. Groundwater samples were collected near the Adyar river in Tamil Nadu, India. Acetamiprid, clothianidin, imidacloprid, thiamethoxam, and dinotefuran standards were purchased from Fisher Scientific. 


\subsection{Methods}

General methods. An Alpha-P instrument (Bruker Instruments) was employed for the attenuated total reflectance Fourier transform infrared analysis (ATR-FTIR) of the dried hydrogels under air. An average of 32 scans was used to generate the infrared spectra. Thermogravimetric analysis (TGA) was performed on a TGA-550 (TA Instruments) with a ramp rate of $20^{\circ} \mathrm{C} \mathrm{min}^{-1}$ from $25{ }^{\circ} \mathrm{C}$ to $800{ }^{\circ} \mathrm{C}$ under $\mathrm{N}_{2}$ constant flow. The NMR measurements were performed on a $400 \mathrm{MHz}$ Bruker Avance III using $\mathrm{CDCl}_{3}$ as a solvent. Possible leaching of GO and polymers from the hydrogels into the water was determined by measuring the total organic carbon (TOC) content of the supernatant, with a high sensitivity Shimadzu TOC-L analyzer having a limit of detection (LOD) of $4 \mu \mathrm{g} \mathrm{L}^{-1}$. The neonicotinoid concentrations were determined using the method reported in [36] under the following conditions: an Agilent 6470B LC/MS/MS triple quadrupole system using a Kromasil C18 column with $5 \mu \mathrm{m}$ particle size, $250 \mathrm{~mm} \times 4.6 \mathrm{~mm}$ dimensions, and $\mathrm{MeCN}: \mathrm{H}_{2} \mathrm{O}$ containing $0.5 \%$ formic acid as mobile phase at a flow rate of $0.5 \mathrm{~mL} \mathrm{~min}^{-1}$ (LOD $0.1 \mu \mathrm{g} \mathrm{L}^{-1}$ ). The molecular mass of PIM-1 was measured by gel permeation chromatography (GPC) set at $1 \mathrm{~mL} \mathrm{~min}^{-1}$ in chloroform using Viscotek VE2001 and TDA302 modules. The nitrogen adsorption $\left(\mathrm{N}_{2}\right)$ isotherms of the powder samples of the three polymers were performed using a surface area and porosimetry analyzer (Micrometrics ASAP 2050) at $-196^{\circ} \mathrm{C}$ up to 1 bar after degassing the samples at $80^{\circ} \mathrm{C}$ for $12 \mathrm{~h}$ with pressure lower than $10 \mu \mathrm{mHg}$. Apparent Brunauer-Emmett-Teller (BET) surface areas were calculated from $\mathrm{N}_{2}$ adsorption data by multi-point BET analysis. The swelling $(\% \mathrm{~S})$ of the hydrogels was determined by measuring the water uptake capacity defined in Eq. 1:

$\% S=100 \frac{m_{w e t}-m_{d r y}}{m_{d r y}}$

Eq. 1

where $\mathrm{m}_{\text {wet }}$ and $\mathrm{m}_{\mathrm{dry}}$ refer to the weights of wet hydrogels at equilibrium and dry hydrogels, respectively.

Synthesis of polymers of intrinsic microporosity (PIM-1). PIM-1 was prepared using an established method [37] that we employed in our previous work [38]. The monomers, $0.01 \mathrm{~mol}$ 5,5',6,6'-tetrahydroxy3,3,3',3'-tetramethyl-1,1'-spirobisindane (TTSBI) and $0.02 \mathrm{~mol}$ tetrafluoroterephthalonitrile (TFTPN), 0.06

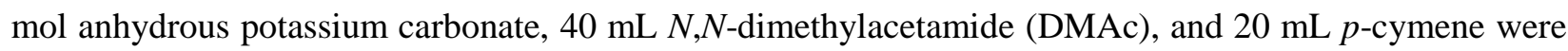
added to a dry reaction flask equipped with a Dean-Stark trap and an IKA overhead stirrer. The reaction conditions were kept anhydrous by performing all steps under argon gas. Once the dissolution was complete, the reaction mixture was refluxed for $30 \mathrm{~min}$. The obtained viscous solution was allowed to precipitate in methanol. The crude product was refluxed for $14 \mathrm{~h}$ in Type II water, followed by washing with $4 \times 80 \mathrm{~mL}$ acetone. The polymer was dried in a vacuum oven at $100{ }^{\circ} \mathrm{C}$ over $24 \mathrm{~h}$. GPC: $M_{\mathrm{n}}=48,500 ; M_{\mathrm{w}}=127,300$; $M_{\mathrm{p}}=87,400 ; M_{\mathrm{w}} / M_{\mathrm{n}}=2.62 .{ }^{1} \mathrm{H} \mathrm{NMR}\left(400 \mathrm{MHz}, \mathrm{CDCl}_{3}\right): \delta 6.71(\mathrm{br}, \mathrm{s}, 2 \mathrm{H}), 6.32(\mathrm{br}, \mathrm{s}, 2 \mathrm{H}), 2.21(\mathrm{br}, \mathrm{s}$, 2H), 2.02 (br, s, 2H), 1.24 (br, s, 6H), 1.26 (br, s, 6H). Surface area: $705 \mathrm{~m}^{2} \mathrm{~g}^{-1}$. Elemental analysis (wt\%) calculated for $\mathrm{C}_{29} \mathrm{H}_{20} \mathrm{~N}_{2} \mathrm{O}_{4}$ molecular formula: $\mathrm{C}, 75.65 \mathrm{H}, 4.35 \mathrm{~N}, 6.09$. Found: $\mathrm{C}, 74.92 \mathrm{H}, 4.41 \mathrm{~N}, 5.87$. 
Hydrogel preparation. The composition of the hydrogels is summarized in Table 1. In a typical procedure to prepare CA/PIM-1/GO beads, $9 \mathrm{~g}$ of cellulose acetate and $0.1 \mathrm{~g}$ PIM-1 were dissolved in a mixture of $100 \mathrm{~mL}$ Cyrene:MeTHF (1:1), followed by the addition of $1 \mathrm{~mL}$ of $1 \mathrm{mg} \mathrm{mL}^{-1} \mathrm{GO}$ suspension. The GO suspension was sonicated for $1 \mathrm{~h}$ prior to use. The mixture of CA/PIM-1/GO suspension was stirred at $300 \mathrm{rpm}$ at $22{ }^{\circ} \mathrm{C}$ for $1 \mathrm{~h}$, followed by the dropwise addition into deionized water as a coagulation medium to yield hydrogels. The hydrogels were kept in water to retain their original morphology.

Table 1. The designations and solid compositions of the hydrogels prepared in Cyrene and MeTHF green solvents. The superscripts and subscripts show the PIM-1 and GO concentrations, respectively.

\begin{tabular}{|c|c|c|c|}
\hline Hydrogel & GO (wt\%) & PIM (wt\%) & CA $(w t \%)$ \\
\hline $\mathbf{H}$ & 0 & 0 & 100 \\
\hline $\mathbf{H}^{\mathrm{PIM} 1}$ & 0 & 1 & 99 \\
\hline $\mathrm{H}_{\mathrm{GO} 0.1}^{\mathrm{PIM} 1}$ & 0.1 & 1 & 98.9 \\
\hline $\mathrm{H}_{\mathrm{GO} 1}^{\mathrm{PIM}} 1$ & 1 & 1 & 98 \\
\hline $\mathrm{H}_{\text {GO } 5}^{\text {PIM 1 }}$ & 5 & 1 & 94 \\
\hline $\mathrm{H}_{\text {GO 10 }}^{\text {PIM 1 }}$ & 10 & 1 & 89 \\
\hline $\mathrm{H}^{\mathrm{PIM} 5}$ & 0 & 5 & 95 \\
\hline $\mathrm{H}^{\text {PIM } 10}$ & 0 & 10 & 90 \\
\hline $\mathrm{H}_{\mathrm{GO} 10}$ & 10 & 0 & 90 \\
\hline
\end{tabular}

SEM analysis. The DI water phase of hydrogels was carefully exchanged to the ethanol phase to minimize the pore collapse. This process was performed at room temperature for $10 \mathrm{~min}$ using 30, 50, 70, 90, and 95\% ethanol/water solutions, step by step. Finally, this process was repeated three times, using $100 \%$ ethanol with the same conditions to make the ethanol-based solution state. Ethanol in the hydrogel was gradually exchanged to carbon dioxide $\left(\mathrm{CO}_{2}\right)$ using the automated critical point dryer (Leica EM CPD300, Leica Microsystems). The dried hydrogels were stored in the desiccator until characterization. Samples were cut using a blade for the cross-sectional imaging and sputter-coated with iridium (thickness of $5 \mathrm{~nm}$ ) to avoid the charging effect. Surface and cross-sectional images of the hydrogels were collected using the scanning electron microscopy (SEM, Teneo, FEI Co., USA). In addition, SEM (Magellan 400 FEG, FEI Co., USA) was used to obtain the high-resolution cross-sectional images of the hydrogels.

XPS analysis. X-ray photoelectron spectroscopy (XPS) experiments were performed on a Kratos Axis Supra instrument equipped with a monochromatic Al Ka X-ray source $(h v=1486.6 \mathrm{eV})$ operated at a power of $150 \mathrm{~W}$ and under UHV conditions in the range of $\sim 10^{-9}$ mbar. All spectra were recorded in the hybrid mode using electrostatic and magnetic lenses and an aperture slot of $300 \mu \mathrm{m} \times 700 \mu \mathrm{m}$. The survey and high-resolution spectra were acquired at fixed analyzer pass energies of $80 \mathrm{eV}$ and $20 \mathrm{eV}$, respectively. The samples were mounted in the floating mode to avoid differential charging. Therefore, XPS spectra were acquired using charge neutralization.

Adsorption isotherms and kinetics. In a typical procedure $1 \mathrm{~L}$ of $1 \mathrm{ppm}$ neonicotinoid (Fig. 2) contaminated water was loaded on 12, 20, 30, 40, 50, 75 and $120 \mathrm{mg}$ hydrogels, the container was sealed, 
and placed in an incubator shaker (KS $4000 \mathrm{i}$ control, IKA) at $23{ }^{\circ} \mathrm{C}$ and $250 \mathrm{rpm}$ for $24 \mathrm{~h}$. Samples from the supernatants were taken to quantify the neonicotinoids. Samples were taken at 0, 0.25, 0.5, 1, 2, 5, 12 , and $24 \mathrm{~h}$ for analysis of the kinetic parameters. The experiments were carried out in duplicates, and the reported errors are standard deviations.

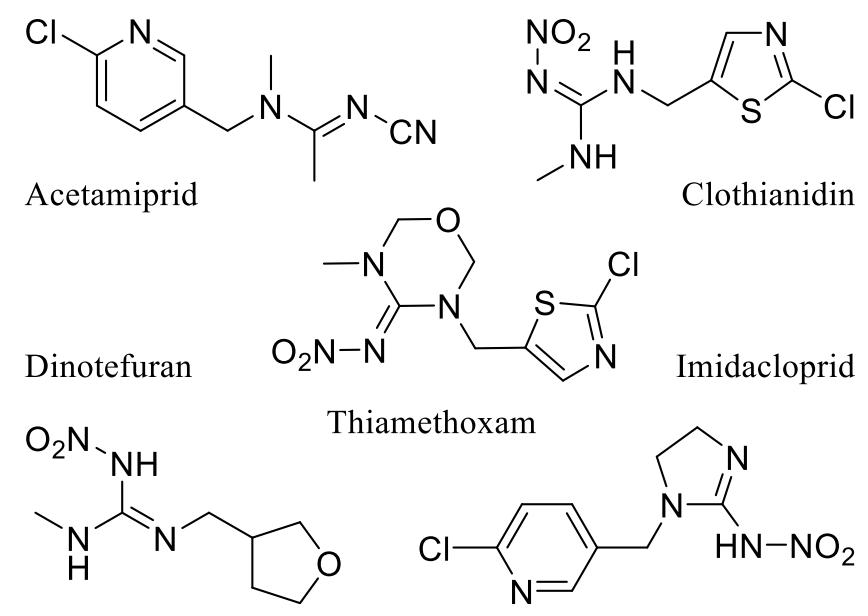

Fig. 2 Chemical structures of neonicotinoids used in this work.

Hydrogel regeneration. Three methods were evaluated for the regeneration of the hydrogels over ten adsorption-desorption cycles, including i) ethanol wash at room temperature, ii) water wash at $40{ }^{\circ} \mathrm{C}$, and iii) water wash using ultrasound. During the adsorption steps, $50 \mathrm{mg}$ hydrogel was stirred with $1 \mathrm{~L}$ of $1 \mathrm{ppm}$ acetamiprid solution for $24 \mathrm{~h}$. During the desorption steps, the hydrogels were filtered, placed in a vial, and stirred at $250 \mathrm{rpm}$ with $3 \times 5 \mathrm{~mL}$ ethanol at $23{ }^{\circ} \mathrm{C}$ or water at $40{ }^{\circ} \mathrm{C}$ in the incubator shaker over $8 \mathrm{~h}$. Alternatively, $3 \times 5 \mathrm{~mL}$ ( $0.5 \mathrm{~min}$ each) water in an Elma P $60 \mathrm{H}$ ultrasonic unit set at $80 \mathrm{kHz}$ frequency and $20 \mathrm{~W}$ power (lowest setting) was used for the desorption over $1.5 \mathrm{~min}$.

Continuous environmental remediation. A process schematic for the continuous adsorption system is shown in Fig. 3. The system consists of two piston pumps, a feed tank containing the contaminated water sample, a regeneration tank containing pure water, two collection tanks for the purified water and the waste stream, and two stainless steel columns (I.D. $\times$ O.D. $\times$ L, $10 \mathrm{~mm} \times 1 / 2^{\prime \prime} \times 25 \mathrm{~cm}$ ) wet-packed with $10 \mathrm{~g}$ of the hydrogel. The hydrogel was soaked in pure water for $24 \mathrm{~h}$ prior to loading the columns. The columns were immersed in Cavitek ultrasonic tanks, which were thermostated at $25^{\circ} \mathrm{C}$. In a typical procedure, the contaminated water and pure water were passed upward through the adsorption and desorption columns at a speed of 10 and $1 \mathrm{~mL} \mathrm{~min}{ }^{-1}$ in an alternating manner, respectively. During each adsorption-desorption cycle, 3.3 L purified water was collected through one of the columns, while the other column was subjected to ultrasound-assisted regenerated using pure water at a flow rate of $1 \mathrm{~mL} \min ^{-1}$ over $1.5 \mathrm{~min}$. Five continuous cycles $(16.5 \mathrm{~L})$ were performed with river water spiked with $10 \mathrm{ppm}$ of acetamiprid, clothianidin, imidacloprid, thiamethoxam, and dinotefuran. The continuous experiment was also performed with $3 \mathrm{~L}$ of locally sourced groundwater from the Adyar river containing 1.31, 0.28, 0.79, and 0.35 ppm of acetamiprid, 
clothianidin, imidacloprid, and thiamethoxam, respectively. Note that dinotefuran was not found in the natural sample. A control experiment was performed on the Type I pure water.

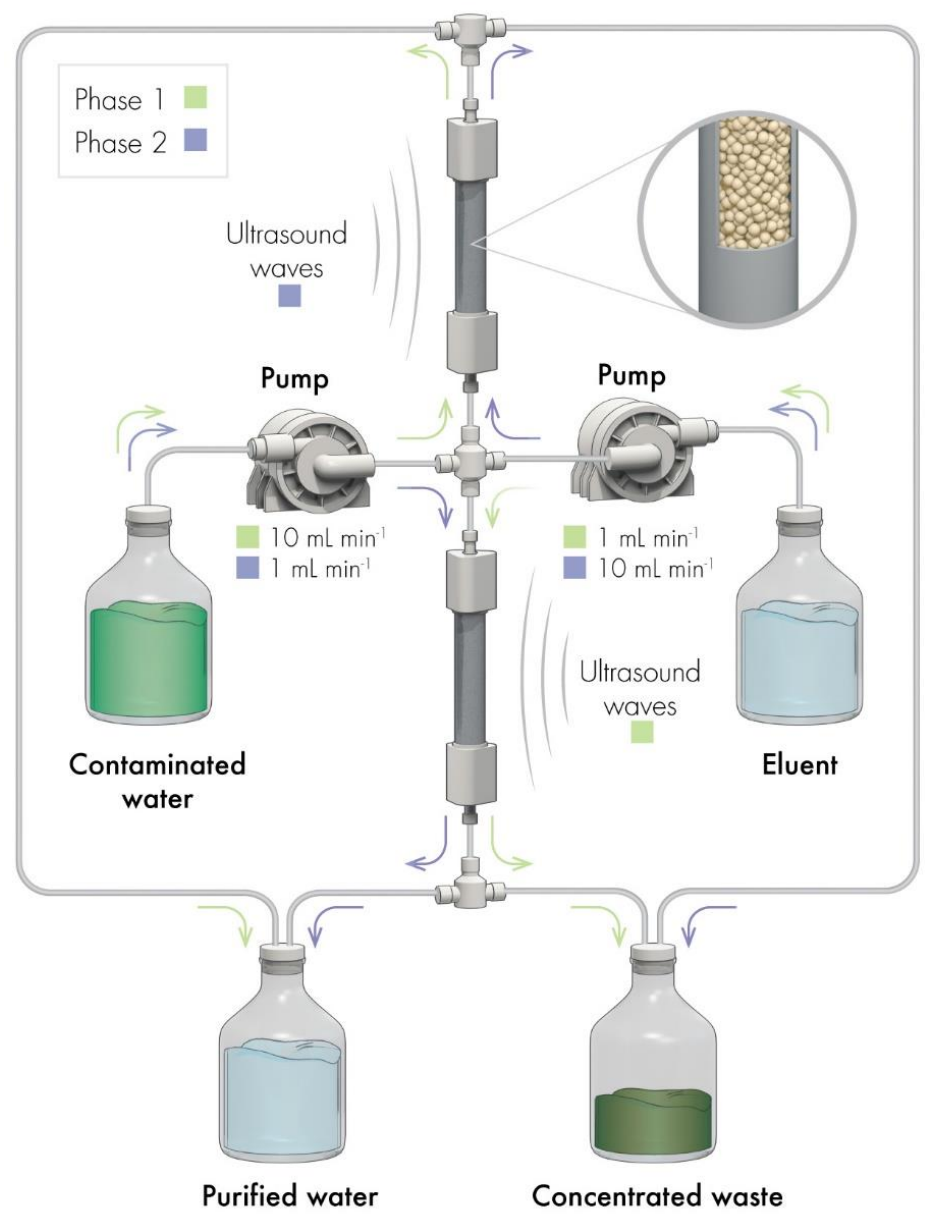

Fig. 3 Illustration of the continuous adsorption and regeneration process for wastewater treatment. Phase 1 refers to adsorption on the top column and desorption on the bottom column, and vice versa for Phase 2 . The 4-way valve in the middle allowed the separation of the purified water and the concentrated waste water into different vessels.

Molecular dynamics. For the four-component model, the periodic $40 \times 30$ supercell of the orthogonal unit cell of graphene-oxide (5376 carbon atoms, 400 epoxy, and 800 hydroxyl groups) was placed into a cubic box with periodic boundary conditions, added two PIM-1 chains of 18 monomers and 500 CA monomers. CA was polymerized using Polymatic [39], and the system was subsequently hydrated. The force field described in references $[39,40]$ was applied for PIM-1, while we used the compatible force field for GO and CA. The system was relaxed using simulated annealing by ten cycles of heating to $600 \mathrm{~K}$ and cooling to 300 $\mathrm{K}$ in 2,000,000 steps each, and finally 10,000,000 steps at constant temperature and constant pressure molecular dynamics simulations at $300 \mathrm{~K}$. The simulations were carried out using the OpenMP parallel version of the LAMMPS simulation package [41]. A time step of $0.9 \mathrm{fs}$ was applied in all cases. Refer to the Supplementary Material for the details of the modeling. 
We analyzed the interactions of the various neonicotinoids with GO using quantum chemical methods. Similarly to the literature [42], we cleaved a circular geometry with diameter of $1.5 \mathrm{~nm}$ from the GO plane and optimized (Fig. S14) together with its neonicotinoid complexes using the GFN2-XTB2 method, specifically developed for the accurate computation of the geometries and non-covalent interaction of large systems [43]. We subsequently computed the interaction energies using the more accurate PBEh-3c/def2mSVP Density Functional method, what is suitable for the accurate computation of the interaction energies [44]. Both the GFN2-XTB and the PBEh-3c methods resulted in similar interaction energies (Table S18).

\section{Results and discussion}

\subsection{Chemical and morphological characterizations}

SEM images of the nanocomposite hydrogels (Fig. 4) show similar core-shell structure for H, $\mathrm{H}^{\mathrm{PIM}} 1$, $\mathrm{H}_{\mathrm{GO} 1}^{\mathrm{PIM} 1}, \mathrm{H}^{\mathrm{PIM} 10}$ and $\mathrm{H}_{\mathrm{GO}} 10$ (Fig. S1). The hydrogel's diameter is around $1.5 \mathrm{~mm}$, which constitutes a thin shell layer with an average thickness of approx. $20 \mathrm{~nm}$. Interestingly, nano-sized beads are formed at the center of the hydrogel core, connected by a fibrous network that hierarchically branches from the middle toward the thin outer layer. We observed similar structures for all nanocomposite hydrogels in this work because the structure formation depends on the phase inversion behavior of the main component, i.e., CA. Regarding thermodynamic and kinetic properties, the highly porous and fibrous network (sponge-like structure) formed beneath the outer shell layer is a result of the slow solvent/non-solvent exchange during the phase inversion [45]. We can reasonably postulate that the CA solution is thermodynamically stable in the presence of hydrogen bonding between the CA and the polar aprotic solvents (Cyrene:MeTHF 1:1), which results in the slow phase inversion rate.
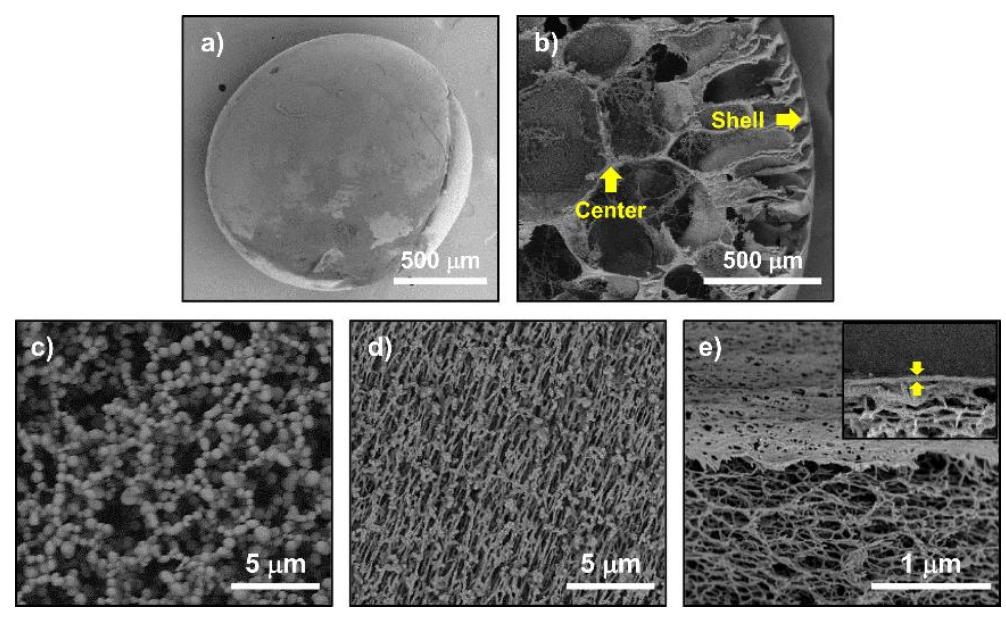

Fig. 4 Typical hydrogel morphology exemplified on $\mathrm{H}_{\mathrm{GO}}{ }_{1}^{\mathrm{PIM}} 1$ through $\mathrm{SEM}$ images showing the (a) whole bead, (b) cross-section, (c) center position, (d) shell position, and (e) near-surface cross-section of the beads. Refer to the Supplementary Material for the morphology of all beads. 
FTIR spectra of the nanocomposite hydrogels are presented in Fig. 5a. The distinctive peaks of GO conflict with those of CA and PIM-1. The stretching vibrations of $\mathrm{O}-\mathrm{H}$ and $\mathrm{C}-\mathrm{H}$ groups appear at approx. $3400 \mathrm{~cm}^{-1}$ and $2900 \mathrm{~cm}^{-1}$, respectively. Strong bands at $1700 \mathrm{~cm}^{-1}$ and $1050 \mathrm{~cm}^{-1}$ correspond to $\mathrm{C}-\mathrm{O}$ and $\mathrm{C}=\mathrm{O}$ stretching vibrations that are present in both $\mathrm{CA}$ and $\mathrm{GO}$. The overall spectra are dominated by the $\mathrm{CA}$ due to the small quantities of PIM-1 and GO in the hydrogels. However, PIM-1 at higher concentrations in the hydrogel can be distinguished within the spectra, as manifested by the slight increase in band intensity at $1410 \mathrm{~cm}^{-1}$, which is attributed to the stretching vibrations of $\mathrm{C}=\mathrm{N}$ [46]. Overall, the similar FTIR spectra for all hydrogels are supported by the XPS analysis, which also confirms the similar structure. The chemical composition of the hydrogels (Fig. 5b, Table S1) demonstrates the dominance of CA as the oxygen and carbon content closely represent the composition of the pristine $\mathrm{CA}(\mathrm{H})$ comprising $\mathrm{C}-\mathrm{OH}, \mathrm{C}=\mathrm{O}$ and $\mathrm{C}-\mathrm{O}$ bonds [47]. There were no significant changes in the chemical composition of the hydrogel surface despite the increase in GO and PIM concentration to $10 \mathrm{wt} \%$ (Fig. S3 and Table S2). These results indicate that the GO and PIM are inside the hydrogel and not on the outer shell layer because XPS only provides the chemical composition of the surface with less than $10 \mathrm{~nm}$ depth.

The TGA curves of the different types of nanocomposite hydrogels are shown in Fig. 5c. The initial weight loss at the temperature range of $50-100{ }^{\circ} \mathrm{C}$ can be attributed to the adsorbed water. Interestingly, the weight loss increased with higher GO loading and decreased with the presence of PIM-1 in the hydrogels. This observation is the result of the abundance of oxygen-containing groups in GO and CA, which resulted in having free water and bound water in the hydrogel network. We observed the characteristic decomposition step of pristine CA and GO-based hydrogels at $150-200{ }^{\circ} \mathrm{C}$ owing to the degradation of the hydroxyl groups, following with a slow decline in weight loss as the temperature increased [48]. Meanwhile, PIM-1 based hydrogels were more stable at temperatures lower than $300^{\circ} \mathrm{C}$ due to the dipole-dipole interactions between polar nitrile groups [49]. The major weight loss for all hydrogels occurred at approx. $355{ }^{\circ} \mathrm{C}$ (Fig. S4, Table S3) due to the breaking of the glycosidic linkage, and subsequently, the degradation of the CA chains [50].

The swelling of the material dictates its ability to capture contaminants efficiently. The difference in osmotic pressure between the solution wicking within the hydrogel and the bulk liquid causes the hydrogels to swell, ultimately leading to structure deterioration depending on the cohesiveness of the material [51]. Very low swelling ratio hydrogels can be more resilient but offer lower swelling capabilities and, therefore, reduced separation performance [52]. We found a linear correlation between the swelling ratio of the hydrogels and the filler content. We generally observed a decrease in swelling behavior as the loading of PIM-1 or GO increased in the hydrogels (Fig. 5d). The effect of GO is more pronounced than PIM-1 in terms of increasing the swelling resistance. The swelling of the CA-based hydrogels decreased from $339 \pm 19 \%$ to $144 \pm 13 \%$ as the PIM-1 content gradually increased to $10 \mathrm{wt} \%$. However, the swelling of hydrogels containing $1 \mathrm{wt} \%$ PIM-1 decreased from $306 \pm 18 \%$ to $86 \pm 13 \%$ as the GO content gradually increased to $5 \mathrm{wt} \%$. This phenomenon is in agreement with the molecular dynamics study detailed in section 3.3, as the GO and CA exhibited a strong interaction via their polar groups, resulting in a tighter network structure, which suggests a prolonged time needed for water to penetrate the material. Thus, a high swelling 
ratio is necessary to ensure exposure of the inner surface of the nanocomposite hydrogels to water and allow the GO to scavenge the neonicotinoids.
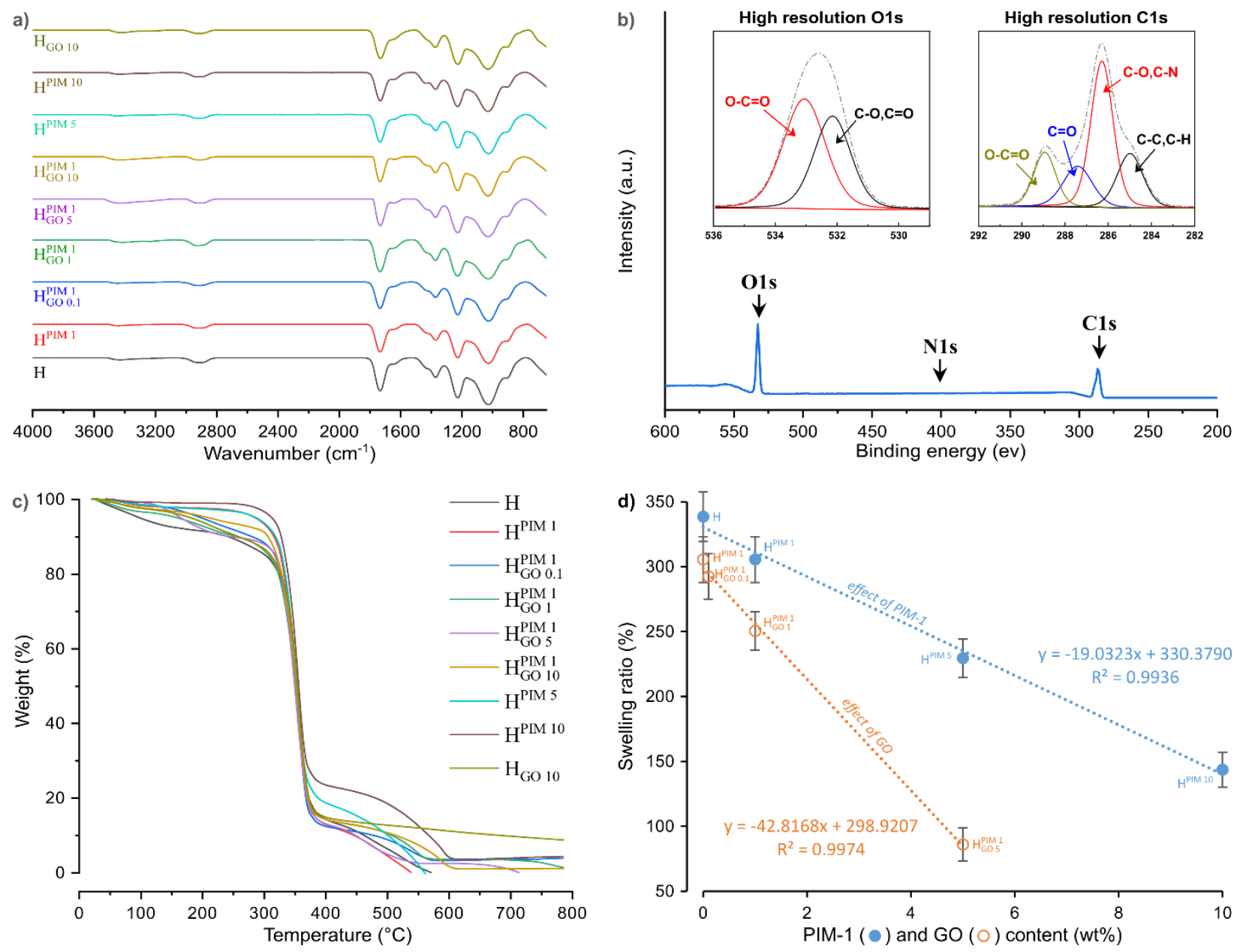

Fig. 5 ATR-FTIR spectra (a), XPS analysis (b), thermogravimetric curves (c), and swelling ratio (d) of the different hydrogels.

\subsection{Neonicotinoid scavenging}

We investigated the neonicotinoid scavenging capability to reveal the effect of PIM-1 and GO by comparing the maximum adsorption capacities and removal efficiencies (Fig. 6a). The adsorption capacity monotonously increased with PIM-1 loading showing a weak performance culminating at $1.8 \mathrm{mg} \mathrm{g}^{-1}$ for $\mathrm{H}^{\mathrm{PIM} 10}$. However, the adsorption capacity significantly improved with the incorporation of $\mathrm{GO}$ in the hydrogel matrix, reaching as high as $20 \mathrm{mg} \mathrm{g}^{-1}$ for $\mathrm{H}_{\mathrm{GO}}^{\mathrm{PIM}} 1$. Interestingly, the high loading of $\mathrm{GO}$ was not sufficient to achieve the same performance, which highlights the importance of the presence of PIM-1. In particular, the adsorption capacity for $\mathrm{H}_{\mathrm{GO}} 10$ without PIM-1 decreased by approx. $82 \%$. We hypothesize that the abundance of the oxygen-containing groups in GO has a high affinity not only for the polar neonicotinoids but also for the CA matrix. However, the incorporation of PIM-1 resulted in a more porous 
structure around the GO sheets, as also shown in the MD simulation in section 3.3, which increased the active surface area of the GO available for scavenging of the neonicotinoids. This assumption is also supported by the observation that the adsorbed neonicotinoid per GO mass decreased with an increase in the GO/PIM-1 ratio. Because the increase in GO content $(1 \rightarrow 5 \rightarrow 10 \mathrm{wt} \%)$ did not significantly enhance the performance of the hydrogel, we selected $\mathrm{H}_{\mathrm{GO}}^{\mathrm{PIM} 1}$ for further investigation.
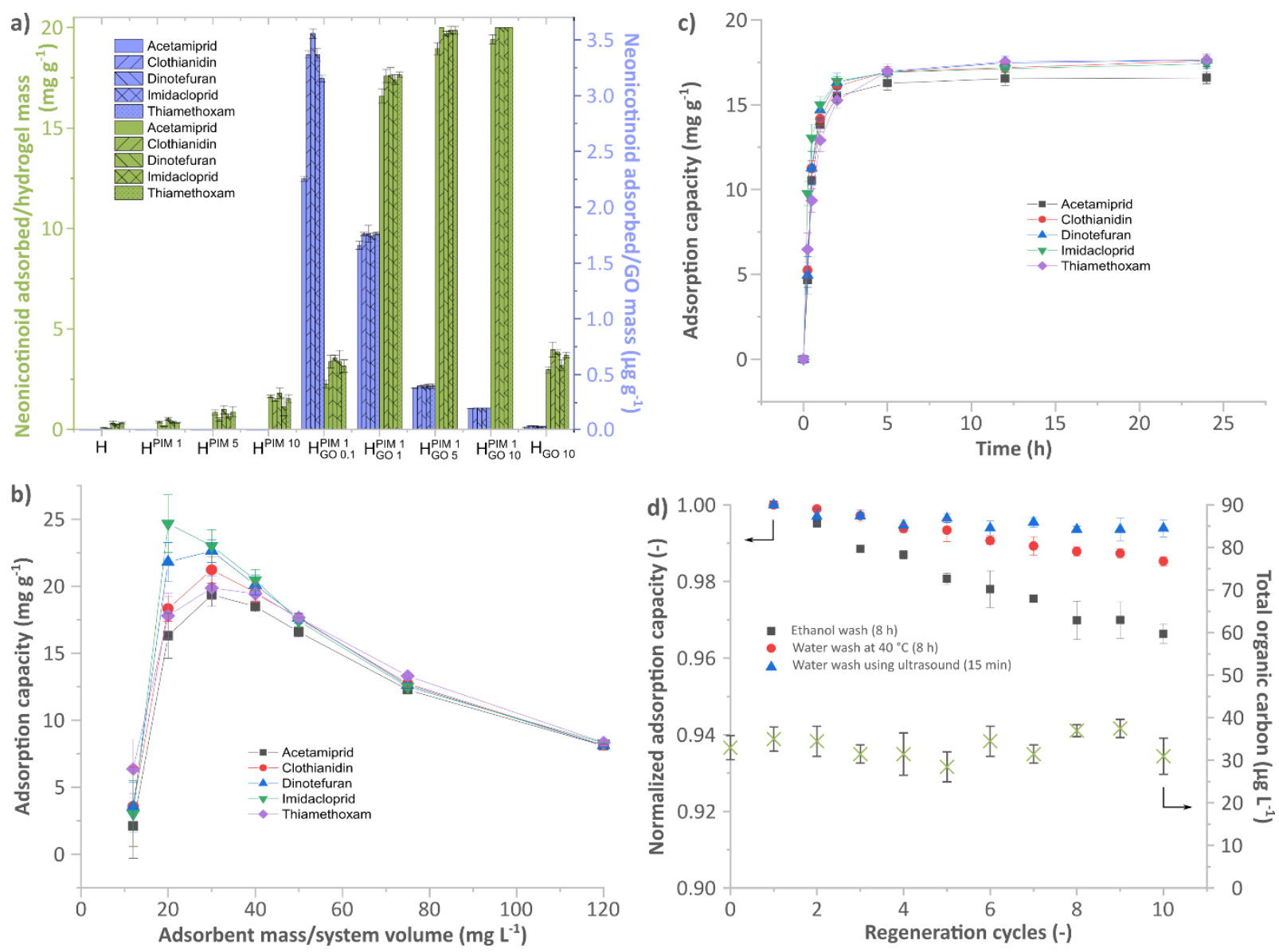

Fig. 6 Hydrogel performance: a) removal efficiency expressed as the amount of neonicotinoid adsorbed per hydrogel or GO mass; b) adsorption isotherms; c) adsorption kinetics at fixed adsorbent mass/system volume ratio of 50 ; d) acetamiprid adsorption capacity and hydrogel components leaching of as a function of $\mathrm{H}_{\mathrm{GO}}{ }_{1}{ }^{1}$ regeneration cycles.

The adsorption data of the neonicotinoids on $\mathrm{H}_{\mathrm{GO}}^{\mathrm{PIM} 1}$ is best fitted using the Redlich-Peterson isotherm compared to other isotherms such as linear, Langmuir, Freundlich, extended Freundlich, reciprocal, Temkin, Sips, Toth, and Jovanovich (Fig. 6b and Table S6). At low concentrations, the adsorption isotherm approaches a Langmuir isotherm. The increase in adsorbent mass should provide more active sites for adsorption to take place. However, the increased mass of $\mathrm{H}_{\mathrm{GO} 1}^{\mathrm{PIM} 1}$ results in a reduction of the adsorption capacity, where Langmuir assumptions are not valid. Such complex adsorption behavior has been reported 
for other nanocomposites used for water treatment [53]. This phenomenon could be explained by the competitive effect between aggregation and adsorption on the hydrogel surface [53,54]. Consequently, the experimental equilibrium data can be fitted with the Redlich-Peterson model by increasing the exponent constant, which concaves the isotherm downward to predict the reduction in the adsorption capacity. This undesirable effect can be avoided by optimizing the ratio of hydrogel mass/system volume to be in the range of 20-50, which allows achieving the highest possible removal efficiency with the least amount of hydrogel $\left(\mathrm{H}_{\mathrm{GO}}^{\mathrm{PIM}} 1\right)$.

Furthermore, the fast kinetic isotherms of $\mathrm{H}_{\mathrm{GO} 1}^{\mathrm{PIM} 1}$ demonstrate its potential for practical applications (Fig. 6c). The rapid adsorption within the first hour was followed by a gradual decrease until reaching the equilibrium plateau after $9 \mathrm{~h}$. Table S14 compares the kinetic behavior with similar works in the literature, which demonstrates the fast kinetics of our hydrogels. The kinetic data were best fitted with Lagergren's pseudo-first-order model, with correlation coefficients higher than 0.99 for all the neonicotinoids. They also achieved the minimum value for the Akaike's Information Criterion (AIC) in comparison with other isotherms such as Ho's pseudo-second-order model, Elovich, and Weber-Morris (Table S7). However, imidacloprid and thiamethoxam fitted exceptionally well the Ho's pseudo-second-order model (e.g., $\mathrm{R}^{2}=0.9986$ for imidacloprid and $\mathrm{R}^{2}=0.9988$ for thiamethoxam). Ho's model best describes chemisorption via electron exchange [55]. Therefore, we hypothesize that the neonicotinoids form various interactions with the GO surface, such as through hydrogen bonding, and $\pi-\pi$ and ionic interactions. The chemisorption mechanism of GO-based nanocomposites has been previously described for the adsorption of dyes and heavy metals in aqueous solutions [56]. All the adsorption and kinetic isotherms are almost identical and within the experimental error, which shows that the chemical class of neonicotinoids have similar adsorption behavior on the nanocomposite hydrogels.

We studied the regeneration and reuse of $\mathrm{H}_{\mathrm{GO}}^{\mathrm{PIM} 1}$ using three different procedures: i) washing with ethanol for $8 \mathrm{~h}$, ii) washing with pure water for $8 \mathrm{~h}$ at $40{ }^{\circ} \mathrm{C}$, and iii) placing in an ultrasound bath with pure water for $15 \mathrm{~min}$ (Fig. 6d). Overall, we found that $\mathrm{H}_{\mathrm{GO}}{ }_{1}^{\mathrm{PIM}} 1$ can be easily regenerated using all the procedures, which preserved at least $96 \%$ of the adsorption capacity, following the reversible characteristics of physisorption. However, the ethanol and the elevated temperature showed a monotonous decrease in performance over the regeneration cycles, and therefore, their application is not recommended from a practical point of view. We found the ultrasound-assisted regeneration to be the most effective procedure in desorbing acetamiprid from $\mathrm{H}_{\mathrm{GO} 1}^{\mathrm{PIM} 1}$ with virtually no performance loss over ten cycles. Employing ultrasound also reduced the time required for regeneration by a factor of 32 compared to the other procedures. This superior performance can be attributed to the simultaneous rarefactions and compressions, or so-called surface cavitation [57], in the hydrogel that, in this case, can break the physical interactions with the acetamiprid.

The morphology of the hydrogel was found to be unaffected by the optimized and repeated short sonication cycles, while long exposure to ultrasound changed the center of the hydrogel from bead-like to fiber-like (Fig. S2). Leaching from nanocomposites such as our hydrogels can jeopardize the purity of the produced water. Thus, we subjected $\mathrm{H}_{\mathrm{GO}}^{\mathrm{PIM} 1} 1$ to a continuous water purification step using Type 1 water to 
investigate potential leaching from the hydrogel. The TOC analysis revealed a constant level of carbon content in the water at $33.2 \pm 3.5 \mu \mathrm{g} \mathrm{L}^{-1}$ concentration (Fig. 6d), which corresponds to the quality of the Type 1 water used. Our findings pave the way for the use of sustainable ultrasound technology for the regeneration of hydrogels, literature reports on which are still scarce (Table S15).

Our nanocomposite hydrogels achieved high removal efficiencies despite operating at a high flow rate $\left(10 \mathrm{~mL} \mathrm{~min}^{-1}\right)$ compared to a $3 \mathrm{~mL} \mathrm{~min}^{-1}$ flow rate reported in the literature [21]. The breakthrough curves of the neonicotinoids during the spiked pure water treatment are shown in Fig. 7a, which are almost identical, as expected based on their adsorption and kinetic isotherms. The breakthrough of neonicotinoids occurred after processing 3.1 L of water. We verified the feasibility of environmental remediation through a continuous adsorption-desorption system for the treatment of contaminated groundwater (Fig. 3). Fig. 7 b reveals the concentration profile over five continuous adsorption-regeneration cycles using two packed bed columns of $\mathrm{H}_{\mathrm{GO} 1}^{\mathrm{PIM} 1}$. One column was used for neonicotinoid scavenging, while the other column was simultaneously regenerated. During each cycle, $3.3 \mathrm{~L}$ of water was purified with a residual neonicotinoid concentration of $1.345 \pm 0.112 \mathrm{ppm}$. The processing of $2.7 \mathrm{~L}$ of water yielded neonicotinoid-free, ultra-pure water with residual neonicotinoid concentration of $0.058 \pm 0.017 \mathrm{ppm}$. Increasing the residual concentration threshold allows for more bed utilization and higher productivity; however, it compromises the purity of the processed water. The source of water affected the bed utilization [58], which was $84 \%$ for the pure water matrix (Fig. 7a) and 70\% for the groundwater matrix (Fig. 7b). These bed utilizations removed 99.9\% of the neonicotinoids. The locally sourced water from the Adyar river had concentrations as low as 1.31, 0.28, 0.79 , and $0.35 \mathrm{ppm}$ of acetamiprid, clothianidin, imidacloprid, and thiamethoxam, respectively. Therefore, we did not observe the breakthrough during the continuous operation with $3 \mathrm{~L}$ of water.
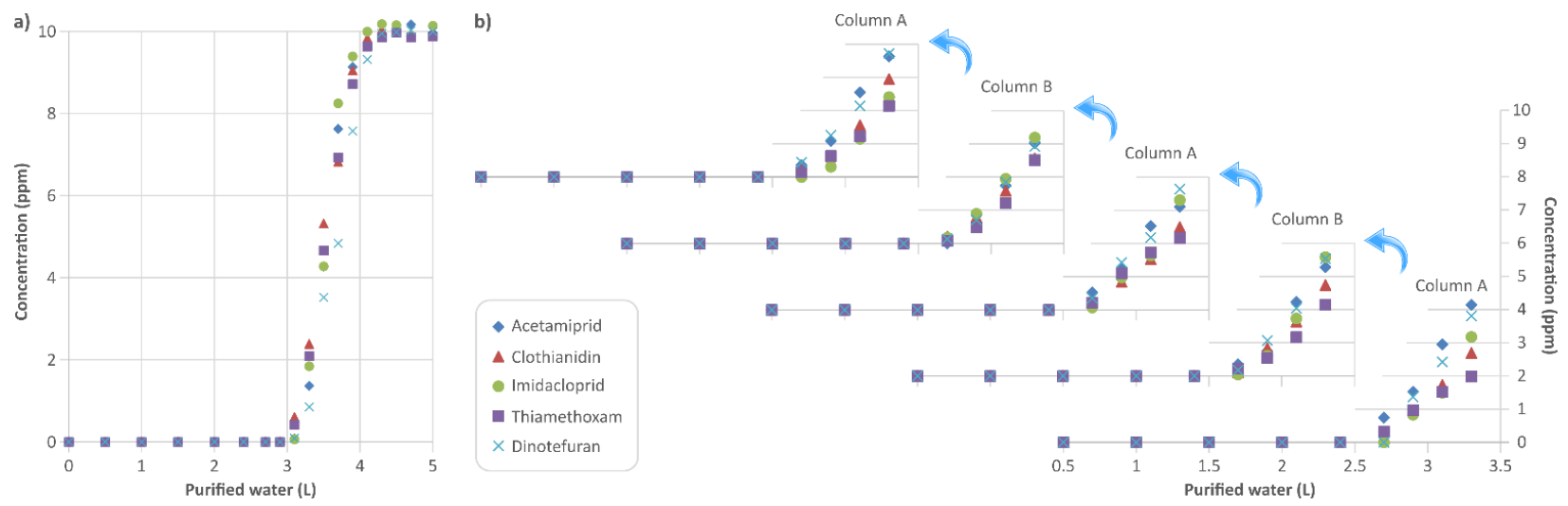

Fig. 7 Continuous packed bed performance: a) breakthrough curve of neonicotinoids on $\mathrm{H}_{\mathrm{GO}} 1_{1}^{\mathrm{PIM}}$; and $\mathrm{b}$ ) the alteration between adsorption and regeneration cycles between columns A and B (see Fig. 3) during the continuous environmental remediation.

We assessed the sustainability of the continuous adsorption process treating contaminated groundwater through the complete (cEF, Eq. 2) and simple (sEF, Eq. 3) E factors, as well as the carbon footprint (Eq. 4), which we found to be $0.10,1.49 \times 10^{-4}$, and $1.99 \mathrm{~kg} \mathrm{~kg}^{-1}$, respectively. This conservative assessment was 
based on the experimentally proven ten regeneration cycles (Fig. 6d). However, regeneration of the hydrogels over 50 cycles improved the sEF by approx. 20\%. Nonetheless, this improvement in cEF is negligible because the concentrated wastewater (desorption cycle) is three orders of magnitude higher than the hydrogel waste. Controlling the temperature of the adsorption process at $25^{\circ} \mathrm{C}$ contributed the most (92.9\%) to the overall carbon footprint. Note that in a field application, a thermostat would not be used, and therefore the carbon footprint would decrease to $0.31 \mathrm{~kg} \mathrm{~kg}^{-1}$. Refer to the Supplementary Material for the green metrics calculations. In general, owing to the ten-fold lower flow rate during the regeneration process (desorption), the yield of the purified water was as high as $90 \%$. Note that the hydrogel regeneration did not require any acid, base, or solvent, and sustainable ultrasound technology was used, with pure water as the eluent. The eluent consumption for the regeneration of the hydrogel was $33 \mathrm{~L} \mathrm{~kg}^{-1}$ cycle ${ }^{-1}$, which is at the lower end for eluent consumption by similar processes (Table S16).

Complete $\mathrm{E}$ factor $(\mathrm{cEF})=\frac{\text { Mass of waste generated including water }(\mathrm{kg})}{\text { Mass of desired product }(\mathrm{kg})}$

Eq. 2

Simple E factor $(\mathrm{sEF})=\frac{\text { Mass of waste generated excluding water }(\mathrm{kg})}{\text { Mass of desired product }(\mathrm{kg})}$

Eq. 3

Carbon footprint $=\frac{\text { Mass of equivalent } \mathrm{CO}_{2}(\mathrm{~kg})}{\text { Mass of desired product }(\mathrm{kg})}$

Eq. 4

\subsection{Revealing the nanoscale interactions}

We computed the maximum pore diameters (corresponding to the largest cross-section of the pore channels), the limiting pore diameters (corresponding to the necks of the pore channels), and the surface areas using the algorithms implemented in the Poreblazer program, as displayed in Table 2. In line with previous studies [40,46], the incorporation of PIM-1 into nanocomposites gives rise to well-defined porous structures. On the contrary, owing to the flexible polymer backbone, the CA without PIM-1 exhibited a nonporous structure with limiting pore diameters smaller than $1.5 \AA$. Water causes the polymers to swell and enlarges the pores; thus, both the limiting and the maximum pore sizes are sensitive to the hydrating conditions. Consequently, the appearance of the pores in the $\mathrm{CA} / \mathrm{H}_{2} \mathrm{O}$ binary system is the consequence of the solvation.

Table 2. Porosity data for binary and ternary mixtures of PIM-1, GO, CA, and $\mathrm{H}_{2} \mathrm{O}$.

\begin{tabular}{cccc}
\hline Mixture & Limiting pore diameter $(\AA)$ & Max. pore diameter $(\AA)$ & Surface area $\left(\mathbf{m}^{\mathbf{2}} \mathbf{g}^{\mathbf{1}}\right)$ \\
\hline $\mathrm{PIM}-1 / \mathrm{GO}$ & 3.7 & 11.7 & 687 \\
$\mathrm{PIM}-1 / \mathrm{H}_{2} \mathrm{O}$ & 8.2 & 14.4 & 1542 \\
$\mathrm{PIM}-1 / \mathrm{GO} / \mathrm{H}_{2} \mathrm{O}$ & 5.0 & 12.4 & 864 \\
$\mathrm{CA} / \mathrm{GO}$ & 1.5 & 4.8 & 0 \\
$\mathrm{CA} / \mathrm{H}_{2} \mathrm{O}$ & 9.6 & 15.9 & 0.67 \\
$\mathrm{CA} / \mathrm{GO} / \mathrm{H}_{2} \mathrm{O}$ & 4.5 & 14.0 & 486 \\
\hline
\end{tabular}


In agreement with the literature [59], the pore structure of the PIM-1/GO system is similar to that of the pure PIM-1 and CA. However, within the PIM-1/GO nanocomposite, pores also exist at the surface of the $\mathrm{GO}$ sheet, which is the result of the rigid structure of PIM-1. However, within the CA/GO nanocomposite, there are no pores near the GO sheet, which can be attributed to two factors: i) CA consists of flexible polymer chains, and ii) owing to the large number of epoxy and hydroxyl groups, the GO strongly interacts with the polar groups of CA. These findings support the experimental observations in Fig. 6a, and explain the role of PIM-1 in increasing the neonicotinoid adsorption on GO.

The geometries and the pore networks for the binary, ternary, and quaternary systems are shown in Fig. 8. The PIM-1/GO/ $\mathrm{H}_{2} \mathrm{O}$ system exhibits several smaller pores, while the pores of $\mathrm{CA} / \mathrm{GO} / \mathrm{H}_{2} \mathrm{O}$ are cumulated into larger pores, in line with the polymer swelling. Therefore, despite the similar pore sizes of $\mathrm{CA} / \mathrm{GO} / \mathrm{H}_{2} \mathrm{O}$ and PIM-1/GO/ $\mathrm{H}_{2} \mathrm{O}$, the surface areas are notably different, as shown in Table 3. In the nanocomposite, the interface between the different materials exhibited several pores, leading to weaker interactions between PIM-1 and GO $\left(-167 \mathrm{~mJ} \mathrm{~m}^{-2}\right)$ than between CA and GO $\left(-408 \mathrm{~mJ} \mathrm{~m}^{-2}\right)$. Due to the numerous hydroxyl and epoxy groups on the GO, such nanosheets offer a partially hydrophilic structure [60]. Consequently, the CA and water compete for adsorption, and the GO sheet is not completely covered by the polymer. These results demonstrate that the pore sizes can be fine-tuned by varying the constituents of the system, which ultimately allows the molecular engineering of nanocomposite hydrogels systems for environmental remediation.

Our investigation of the PIM-1/GO/CA/ $\mathrm{H}_{2} \mathrm{O}$ quaternary system (Fig. 8h-k) revealed that the $\mathrm{CA} / \mathrm{GO}$ interaction is more favored than that of the $\mathrm{PIM}-1 / \mathrm{GO}$; in particular, the shorter $\mathrm{CA}$ chains prefer the interaction with the GO plane and flattening on the surface. Therefore, larger molecular weight CA is recommended for adsorptive hydrogels. Because of the swelling, filaments consisting of CA chains can be found between the replicas of the GO plane, i.e., the CA holds the nanocomposite together. We observed that a few PIM-1 attach directly to the GO sheet, which are surrounded by water and flexible CA chains. Hence, PIM-1 provides porosity to the nanocomposite locally at the GO surface and therefore makes the GO available for scavenging neonicotinoids.

The results in Fig. 81 shows that all the investigated neonicotinoids interact favorably with GO, with relatively small variation in the interaction energies (-75 to $\left.-91 \mathrm{~kJ} \mathrm{~mol}^{-1}\right)$, except for acetamiprid (-143 $\mathrm{kJ}$ $\mathrm{mol}^{-1}$ ). In agreement with the literature [42], the hydrogen bond interactions between the hydroxyl and epoxy groups of the GO and the polar groups of the neonicotinoids are the most important for their interactions. The favorable hydrogen-bonding explains the increased stability of the GO-acetamiprid complex. 
a)

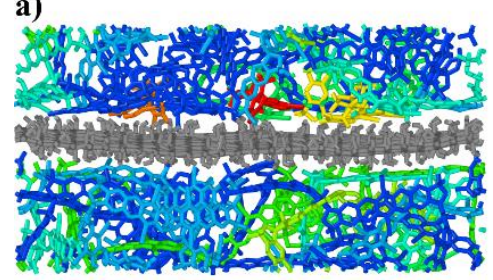

d)

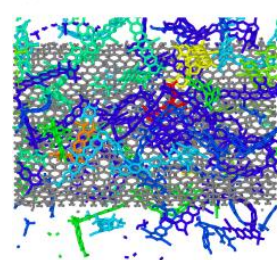

h)

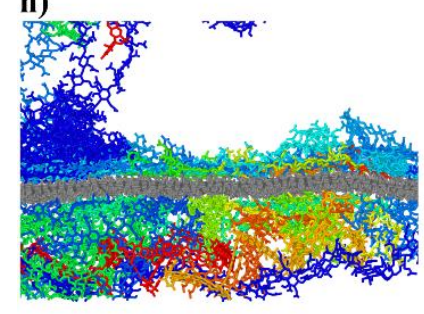

b)

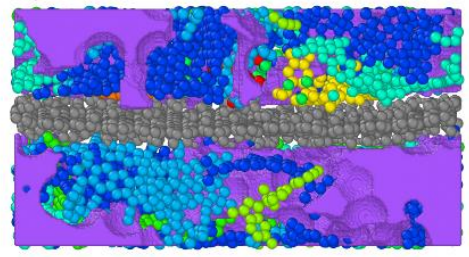

f)

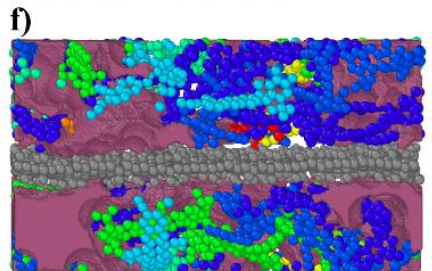

i)

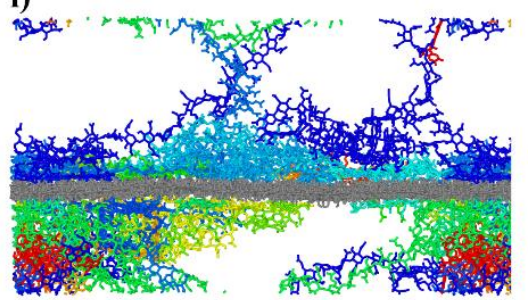

c)

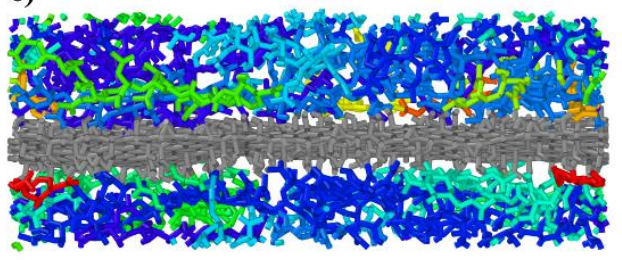

g) l)

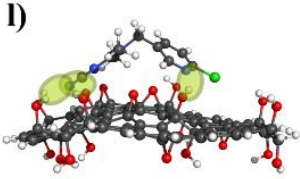

Acetamiprid: $-143 \mathrm{~kJ} \mathrm{~mol}^{-1}$

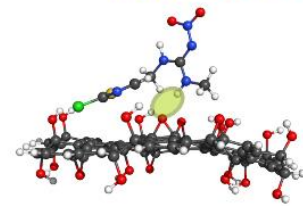

Clothiamidin: $-78 \mathrm{~kJ} \mathrm{~mol}^{-1}$

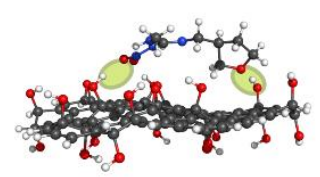

Dinotefuran: $-87 \mathrm{~kJ} \mathrm{~mol}^{-1}$ j)

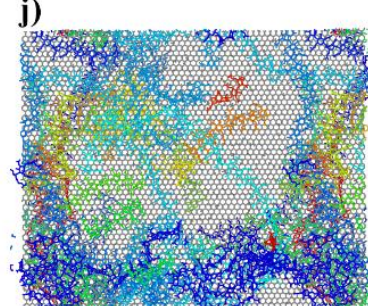

k)

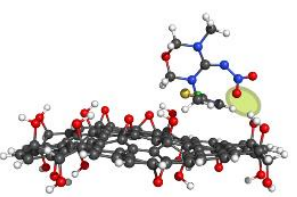

Thiamethoxam: $-75 \mathrm{~kJ} \mathrm{~mol}^{-1}$
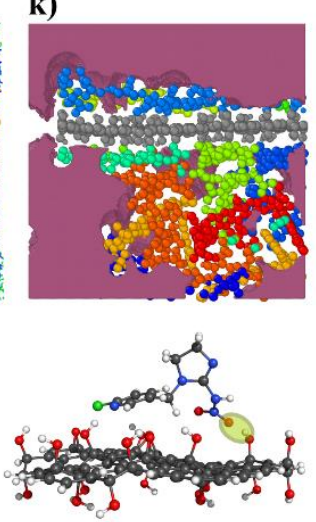

Imidacloprid: $-91 \mathrm{~kJ} \mathrm{~mol}^{-1}$

Fig. 8. Geometrical structures and pore networks. PIM-1/GO binary system in the dry state showing the geometries (a) and pore network (b) for the same orientation. PIM-1/CA binary system in the dry state showing the geometries (c). PIM-1/GO/ $\mathrm{H}_{2} \mathrm{O}(\mathrm{d}, \mathrm{f})$ and $\mathrm{CA} / \mathrm{GO} / \mathrm{H}_{2} \mathrm{O}(\mathrm{e}, \mathrm{g})$ ternary systems in the solvated state showing the geometries (d,e) and pore networks (f,g) for the same orientation. Geometric structures of the PIM-1/GO/CA/ $\mathrm{H}_{2} \mathrm{O}$ quaternary system from the side $(\mathrm{h}, \mathrm{i})$ and top $(\mathrm{j})$ views. Pore network $(\mathrm{k})$ of a representative part of the quaternary system: the CA strongly attached to the top of the GO sheet, while both PIM-1 and CA are present below the GO sheet, providing a ticker but more porous structure. The pores are filled with water in the simulation. The GO is depicted in gray, the polymers are colored according to the different chains, and the pores are shown in purple. Interaction energy (1) of the GO with the neonicotinoids, where the hydrogen bonds are highlighted with green ellipses on the molecular geometries.

\section{Conclusions}

We successfully produced nanocomposite hydrogels prepared from green solvents such as Cyrene and MeTHF and composed of sustainable cellulose acetate, and $0.1-10 \mathrm{wt} \%$ polymers of intrinsic microporosity (PIM-1), and graphene oxide (GO). The structure and pore networks were simulated using empirical force field-based molecular dynamics simulations that revealed the synergistic effects of PIM-1 and GO through the increase in surface area available for neonicotinoid scavenging. We observed high adsorption capacity culminating at $20 \mathrm{mg} \mathrm{g}^{-1}$ and fast kinetic behavior towards acetamiprid, clothianidin, dinotefuran, imidacloprid, and thiamethoxam. Continuous environmental remediation of contaminated groundwater from the Adyar river resulted in concentrations of neonicotinoid pollution of less than $0.1 \mu \mathrm{g} \mathrm{L}^{-1}$. The yield 
of purified water was as high as $90 \%$. We demonstrated the sustainable ultrasound-assisted regeneration of the scavengers over ten cycles with virtually $100 \%$ recovery of the adsorption capacity. The presented methodology is expandable to other contaminants in both aqueous environments and organic media.

\section{Acknowledgment}

The graphical abstract and Figures 1 and 3 were created by Xavier Pita, scientific illustrator at King Abdullah University of Science and Technology (KAUST). AA acknowledges the PhD scholarship from Saudi Aramco. We thank Ali Reza Behzad from the Imaging and Characterization Core Lab at KAUST for assisting with the cryo-SEM measurements. The research reported in this publication was supported by funding from KAUST. TH thanks the Hungarian Government and the European Union, Grant/Award Number: VEKOP-2.1.1-15-2016-00114 for their support.

\section{Data availability}

The data that support the findings of this study is available in the Supplementary Material, and available from the corresponding author upon request.

\section{References}

[1] FAO, World Food and Agriculture - Statistical Pocketbook, Rome, 2018.

[2] C. Bass, I. Denholm, M.S. Williamson, R. Nauen, The global status of insect resistance to neonicotinoid insecticides, Pestic. Biochem. Physiol. 121 (2015) 78-87. https://doi.org/10.1016/j.pestbp.2015.04.004.

[3] C.A. Morrissey, P. Mineau, J.H. Devries, F. Sanchez-Bayo, M. Liess, M.C. Cavallaro, K. Liber, Neonicotinoid contamination of global surface waters and associated risk to aquatic invertebrates: A review, Environ. Int. 74 (2015) 291-303. https://doi.org/10.1016/j.envint.2014.10.024.

[4] F. Sanchez-Bayo, The trouble with neonicotinoids, Science. 346 (2014) 806-807. https://doi.org/10.1126/science.aan3684.

[5] M.L. Hladik, A.R. Main, D. Goulson, Environmental Risks and Challenges Associated with Neonicotinoid Insecticides, Environ. Sci. Technol. 52 (2018) 3329-3335. https://doi.org/10.1021/acs.est.7b06388.

[6] EASAC, Ecosystem services, agriculture and neonicotinoids, 2015.

[7] N. Tsvetkov, O. Samson-Robert, K. Sood, H.S. Patel, D.A. Malena, P.H. Gajiwala, P. Maciukiewicz, V. Fournier, A. Zayed, Chronic exposure to neonicotinoids reduces honey bee health near corn crops, Science. 356 (2017) 1395-1397. https://doi.org/10.1126/science.aam7470.

[8] B.A. Woodcock, N.J.B. Isaac, J.M. Bullock, D.B. Roy, D.G. Garthwaite, A. Crowe, R.F. Pywell, Impacts of neonicotinoid use on long-term population changes in wild bees in England, Nat. Commun. 7 (2016) 12459. https://doi.org/10.1038/ncomms12459.

[9] M. Rundlöf, G.K.S. Andersson, R. Bommarco, I. Fries, V. Hederström, L. Herbertsson, O. Jonsson, B.K. Klatt, T.R. Pedersen, J. Yourstone, H.G. Smith, Seed coating with a neonicotinoid insecticide negatively affects wild bees, Nature. 521 (2015) 77-80. 
https://doi.org/10.1038/nature14420.

[10] F. Sanchez-Bayo, K. Goka, Pesticide residues and bees - A risk assessment, PLoS One. 9 (2014) e94482. https://doi.org/10.1371/journal.pone.0094482.

[11] N.E. Raine, R.J. Gill, Ecology: Tasteless pesticides affect bees in the field, Nature. 521 (2015) 3839. https://doi.org/10.1038/nature14391.

[12] D. Butler, Scientists hail European ban on bee-harming pesticides, Nature. (2018). https://doi.org/10.1038/d41586-018-04987-4.

[13] E. Stokstad, European agency concludes controversial 'neonic' pesticides threaten bees, Science (80-. ). (2018). https://doi.org/10.1126/science.aat4598.

[14] Z. Chen, X. Yao, F. Dong, H. Duan, X. Shao, X. Chen, T. Yang, G. Wang, Y. Zheng, Ecological toxicity reduction of dinotefuran to honeybee: New perspective from an enantiomeric level, Environ. Int. 130 (2019) 104854. https://doi.org/10.1016/j.envint.2019.05.048.

[15] E.A.D. Mitchell, B. Mulhauser, M. Mulot, A. Mutabazi, G. Glauser, A. Aebi, A worldwide survey of neonicotinoids in honey, Science. 358 (2017) 109-111. https://doi.org/10.1126/science.aan3684.

[16] R. Cernansky, Controversial pesticides found in honey samples from six continents, Nature. (2017). https://doi.org/10.1038/nature.2017.22762.

[17] H.A. Craddock, D. Huang, P.C. Turner, L. Quirós-Alcalá, D.C. Payne-Sturges, Trends in neonicotinoid pesticide residues in food and water in the United States, 1999-2015, Environ. Heal. A Glob. Access Sci. Source. 18 (2019) 7. https://doi.org/10.1186/s12940-018-0441-7.

[18] D. Chen, Y. Zhang, B. Lv, Z. Liu, J. Han, J. Li, Y. Zhao, Y. Wu, Dietary exposure to neonicotinoid insecticides and health risks in the Chinese general population through two consecutive total diet studies, Environ. Int. 135 (2020) 105399. https://doi.org/10.1016/j.envint.2019.105399.

[19] D.A. Thompson, H.-J. Lehmler, D.W. Kolpin, M.L. Hladik, J.D. Vargo, K.E. Schilling, G.H. LeFevre, T.L. Peeples, M.C. Poch, L.E. LaDuca, D.M. Cwiertny, R.W. Field, A critical review on the potential impacts of neonicotinoid insecticide use: current knowledge of environmental fate, toxicity, and implications for human health, Environ. Sci. Process. Impacts. (2020) Advance Article. https://doi.org/10.1039/c9em00586b.

[20] L. Weerasundara, B. Gabriele, A. Figoli, Y.S. Ok, J. Bundschuh, Hydrogels: Novel materials for contaminant removal in water-A review, Crit. Rev. Environ. Sci. Technol. (2020). https://doi.org/10.1080/10643389.2020.1776055.

[21] X. Song, J. An, C. He, J. Zhou, Y. Xu, H. Ji, L. Yang, J. Yin, W. Zhao, C. Zhao, A bioinspired strategy towards super-adsorbent hydrogel spheres: Via self-sacrificing micro-reactors for robust wastewater remediation, J. Mater. Chem. A. 7 (2019) 21386-21403. https://doi.org/10.1039/c9ta05550a.

[22] L. Wang, G. Yu, J. Li, Y. Feng, Y. Peng, X. Zhao, Y. Tang, Q. Zhang, Stretchable hydrophobic modified alginate double-network nanocomposite hydrogels for sustained release of waterinsoluble pesticides, J. Clean. Prod. 226 (2019) 122-132. https://doi.org/10.1016/j.jclepro.2019.03.341.

[23] Y. Xiang, G. Zhang, C. Chen, B. Liu, D. Cai, Z. Wu, Fabrication of a pH-Responsively ControlledRelease Pesticide Using an Attapulgite-Based Hydrogel, ACS Sustain. Chem. Eng. 6 (2018) 11921201. https://doi.org/10.1021/acssuschemeng.7b03469.

[24] X. Hu, L. Yan, Y. Wang, M. Xu, Freeze-thaw as a route to build manageable polysaccharide cryogel for deep cleaning of crystal violet, Chem. Eng. J. 396 (2020) 125354. https://doi.org/10.1016/j.cej.2020.125354.

[25] X. Hu, L. Yan, Y. Wang, M. Xu, Self-assembly of binary oppositely charged polysaccharides into 
polyelectrolyte complex hydrogel film for facile and efficient Pb2+ removal, Chem. Eng. J. 388 (2020) 124189. https://doi.org/10.1016/j.cej.2020.124189.

[26] Y. Zhuang, F. Yu, H. Chen, J. Zheng, J. Ma, J. Chen, Alginate/graphene double-network nanocomposite hydrogel beads with low-swelling, enhanced mechanical properties, and enhanced adsorption capacity, J. Mater. Chem. A. 4 (2016) 10885-10892. https://doi.org/10.1039/c6ta02738e.

[27] Y. Zhuang, Q. Liu, Y. Kong, C. Shen, H. Hao, D.D. Dionysiou, B. Shi, Enhanced antibiotic removal through a dual-reaction-center Fenton-like process in 3D graphene based hydrogels, Environ. Sci. Nano. 6 (2019) 388-398. https://doi.org/10.1039/C8EN01339J.

[28] M.N. Alnajrani, O.A. Alsager, Removal of Antibiotics from Water by Polymer of Intrinsic Microporosity: Isotherms, Kinetics, Thermodynamics, and Adsorption Mechanism, Sci. Rep. 10 (2020) 794. https://doi.org/10.1038/s41598-020-57616-4.

[29] F. Topuz, M.A. Abdulhamid, S.P. Nunes, G. Szekely, Hierarchically porous electrospun nanofibrous mats produced from intrinsically microporous fluorinated polyimide for the removal of oils and non-polar solvents, Environ. Sci. Nano. 7 (2020) 1365-1372. https://doi.org/10.1039/d0en00084a.

[30] J. Byun, H.A. Patel, D. Thirion, C.T. Yavuz, Charge-specific size-dependent separation of watersoluble organic molecules by fluorinated nanoporous networks, Nat. Commun. 7 (2016) 13377. https://doi.org/10.1038/ncomms13377.

[31] K.Q. Lu, X. Xin, N. Zhang, Z.R. Tang, Y.J. Xu, Photoredox catalysis over graphene aerogelsupported composites, J. Mater. Chem. A. 6 (2018) 4590-4604. https://doi.org/10.1039/c8ta00728d.

[32] Y. Chen, X. Xie, X. Xin, Z.R. Tang, Y.J. Xu, $\mathrm{Ti}_{3} \mathrm{C}_{2} \mathrm{~T}_{\mathrm{x}}$-Based three-dimensional hydrogel by a graphene oxide-assisted self-convergence process for enhanced photoredox catalysis, ACS Nano. 13 (2019) 295-304. https://doi.org/10.1021/acsnano.8b06136.

[33] Z. Chen, J. Wang, X. Duan, Y. Chu, X. Tan, S. Liu, S. Wang, Facile fabrication of 3D ferrous ion crosslinked graphene oxide hydrogel membranes for excellent water purification, Environ. Sci. Nano. 6 (2019) 3060-3071. https://doi.org/10.1039/c9en00638a.

[34] Y. Sun, F. Yu, C. Li, X. Dai, J. Ma, Nano-/Micro-confined Water in Graphene Hydrogel as Superadsorbents for Water Purification, Nano-Micro Lett. 12 (2020). https://doi.org/10.1007/s40820-019-0336-3.

[35] P.C. Bandara, J.V.D. Perez, E.T. Nadres, R.G. Nannapaneni, K.J. Krakowiak, D.F. Rodrigues, Graphene Oxide Nanocomposite Hydrogel Beads for Removal of Selenium in Contaminated Water, ACS Appl. Polym. Mater. 1 (2019) 2668-2679. https://doi.org/10.1021/acsapm.9b00612.

[36] A. Suganthi, K. Bhuvaneswari, M. Ramya, Determination of neonicotinoid insecticide residues in sugarcane juice using LCMSMS, Food Chem. 241 (2018) 275-280. https://doi.org/10.1016/j.foodchem.2017.08.098.

[37] N. Du, G.P. Robertson, J. Song, I. Pinnau, S. Thomas, M.D. Guiver, Polymers of intrinsic microporosity containing trifluoromethyl and phenylsulfone groups as materials for membrane gas separation, Macromolecules. 41 (2008) 9656-9662. https://doi.org/10.1021/ma801858d.

[38] M. Razali, J.F. Kim, M. Attfield, P.M. Budd, E. Drioli, Y.M. Lee, G. Szekely, Sustainable wastewater treatment and recycling in membrane manufacturing, Green Chem. 17 (2015) 51965205. https://doi.org/10.1039/c5gc01937k.

[39] L.J. Abbott, K.E. Hart, C.M. Colina, Polymatic: A generalized simulated polymerization algorithm for amorphous polymers, Theor. Chem. Acc. 132 (2013) 1334. https://doi.org/10.1007/s00214013-1334-z. 
[40] G.S. Larsen, P. Lin, K.E. Hart, C.M. Colina, Molecular simulations of pim-1-like polymers of intrinsic microporosity, Macromolecules. 44 (2011) 6944-6951. https://doi.org/10.1021/ma200345v.

[41] S. Plimpton, Fast parallel algorithms for short-range molecular dynamics, J. Comput. Phys. 117 (1995) 1-19. https://doi.org/10.1006/jcph.1995.1039.

[42] H. Vovnteraction of nucleobases and aromatic amino acids with grapusha, S. Sanyal, B. Sanyal, Interaction of nucleobases and aromatic amino acids with graphene oxide and graphene flakes, $\mathrm{J}$. Phys. Chem. Lett. 4 (2013) 3710-3718. https://doi.org/10.1021/jz401929h.

[43] C. Bannwarth, S. Ehlert, S. Grimme, GFN2-xTB - An Accurate and broadly parametrized selfconsistent tight-binding quantum chemical method with multipole electrostatics and densitydependent dispersion contributions, J. Chem. Theory Comput. 15 (2019) 1652-1671. https://doi.org/10.1021/acs.jctc.8b01176.

[44] S. Grimme, J.G. Brandenburg, C. Bannwarth, A. Hansen, Consistent structures and interactions by density functional theory with small atomic orbital basis sets, J. Chem. Phys. 143 (2015) 054107. https://doi.org/10.1063/1.4927476.

[45] S. Li, Z. Cui, L. Zhang, B. He, J. Li, The effect of sulfonated polysulfone on the compatibility and structure of polyethersulfone-based blend membranes, J. Membr. Sci. 513 (2016) 1-11. https://doi.org/10.1016/j.memsci.2016.04.035.

[46] N. Du, H.B. Park, G.P. Robertson, M.M. Dal-Cin, T. Visser, L. Scoles, M.D. Guiver, Polymer nanosieve membranes for CO2-capture applications, Nat. Mater. 10 (2011) 372-375. https://doi.org/10.1038/nmat2989.

[47] A. Jayalakshmi, I.C. Kim, Y.N. Kwon, Cellulose acetate graft-(glycidylmethacrylate-g-PEG) for modification of AMC ultrafiltration membranes to mitigate organic fouling, RSC Adv. 5 (2015) 48290-48300. https://doi.org/10.1039/c5ra03499j.

[48] M. Kumar, A.M. Isloor, T. Somasekhara Rao, A.F. Ismail, R. Farnood, P.M.G. Nambissan, Removal of toxic arsenic from aqueous media using polyphenylsulfone/cellulose acetate hollow fiber membranes containing zirconium oxide, Chem. Eng. J. 393 (2020) 124367. https://doi.org/10.1016/j.cej.2020.124367.

[49] F.Y. Li, Y. Xiao, T.S. Chung, S. Kawi, High-performance thermally self-cross-linked polymer of intrinsic microporosity (PIM-1) membranes for energy development, Macromolecules. 45 (2012) 1427-1437. https://doi.org/10.1021/ma202667y.

[50] Z.Q. Liu, A.M. Cunha, X.S. Yi, C.A. Bernardo, Thermal characterizations of wood flour/starch cellulose acetate compounds, J. Macromol. Sci. - Phys. 40 (2001) 529-538. https://doi.org/10.1081/MB-100106175.

[51] H. Kamata, Y. Akagi, Y. Kayasuga-Kariya, U. Il Chung, T. Sakai, "Nonswellable" hydrogel without mechanical hysteresis, Science (80-. ). 343 (2014) 873-875. https://doi.org/10.1126/science.1247811.

[52] Q. Lv, X. Hu, X. Zhang, L. Huang, Z. Liu, G. Sun, Highly efficient removal of trace metal ions by using poly(acrylic acid) hydrogel adsorbent, Mater. Des. 181 (2019) 107934. https://doi.org/10.1016/j.matdes.2019.107934.

[53] A.M. Mohammad, T.A. Salah Eldin, M.A. Hassan, B.E. El-Anadouli, Efficient treatment of leadcontaining wastewater by hydroxyapatite/chitosan nanostructures, Arab. J. Chem. 10 (2017) 683690. https://doi.org/10.1016/j.arabjc.2014.12.016.

[54] P. Linse, H. Wennerström, Adsorption versus aggregation. Particles and surface of the same material, Soft Matter. 8 (2012) 2486-2493. https://doi.org/10.1039/c2sm07000f.

[55] Y.S. Ho, Review of second-order models for adsorption systems, J. Hazard. Mater. B136 (2006) 
681-689. https://doi.org/10.1016/j.jhazmat.2005.12.043.

[56] J. Xiong, D. Zhang, H. Lin, Y. Chen, Amphiprotic cellulose mediated graphene oxide magnetic aerogels for water remediation, Chem. Eng. J. 400 (2020) 125890.

https://doi.org/10.1016/j.cej.2020.125890.

[57] H. Daghooghi-Mobarakeh, N. Campbell, W.K. Bertrand, P.G. Kumar, S. Tiwari, L. Wang, R. Wang, M. Miner, P.E. Phelan, Ultrasound-assisted regeneration of zeolite/water adsorption pair, Ultrason. Sonochem. 64 (2020) 105042. https://doi.org/10.1016/j.ultsonch.2020.105042.

[58] F. Li, A. Yuasa, K. Ebie, Y. Azuma, T. Hagishita, Y. Matsui, Factors affecting the adsorption capacity of dissolved organic matter onto activated carbon: Modified isotherm analysis, Water Res. 36 (2002) 4592-4604. https://doi.org/10.1016/S0043-1354(02)00174-4.

[59] A. Gonciaruk, K. Althumayri, W.J. Harrison, P.M. Budd, F.R. Siperstein, PIM-1/graphene composite: A combined experimental and molecular simulation study, Microporous Mesoporous Mater. 209 (2015) 126-134. https://doi.org/10.1016/j.micromeso.2014.07.007.

[60] B. Lian, S. De Luca, Y. You, S. Alwarappan, M. Yoshimura, V. Sahajwalla, S.C. Smith, G. Leslie, R.K. Joshi, Extraordinary water adsorption characteristics of graphene oxide, Chem. Sci. 9 (2018) 5106-5111. https://doi.org/10.1039/c8sc00545a. 